\title{
Questes
}

\section{Figures de l'autorité : éléments bibliographiques}

\author{
Julien Abed
}

\section{OpenEdition}

\section{Journals}

\section{Édition électronique}

URL : http://journals.openedition.org/questes/1612

DOI : 10.4000/questes. 1612

ISSN : 2109-9472

\section{Éditeur}

Les Amis de Questes

\section{Édition imprimée}

Date de publication : 15 janvier 2006

Pagination : 65-69

ISSN : 2102-7188

\section{Référence électronique}

Julien Abed, « Figures de l'autorité : éléments bibliographiques », Questes [En ligne], 8 | 2006, mis en ligne le 01 janvier 2014, consulté le 15 septembre 2020. URL : http://journals.openedition.org/ questes/1612

Ce document a été généré automatiquement le 15 septembre 2020.

(C) Association des amis de «Questes » 


\title{
Figures de l'autorité : éléments bibliographiques
}

\author{
Julien Abed
}

AlLIROT, Anne-Hélène, LECUPPRE, Gilles et SCORDIA, Lydwine (éds.), Royautés imaginaires (XII ${ }^{e}-\mathrm{VVI}^{e}$ siècles), actes du colloque organisé par le Centre de Recherche d'Histoire sociale et culturelle de l'université de Paris X-Nanterre (26-27 septembre 2003), Turnhout, Brepols (coll. «Culture et société médiévales »), 2005.

ANCONA, Elvio, All'origine della sovranità : sistema gerarchico e ordinamento giuridico nella disputa sui due poteri all'inizio del XIV secolo, Elvio Ancona, Torino, G. Giappichelli, 2004.

Auctor et auctoritas: invention et conformisme dans l'écriture médiévale, Actes du colloque tenu à l'Université de Versailles-Saint-Quentin-en-Yvelines, 14-16 juin 1999, réunis sous la dir. de Michel zimMERMAnN, Paris, École des chartes, 2001.

Auctoritas, Mélanges offerts au professeur Olivier Guillot, études réunies par Giles CONSTABLE et Michel Rouche, Paris, Presses universitaires de la Sorbonne, 2006.

L'autorité de l'Écriture, Actes du colloque, septembre 2000, Jérusalem, organisé par l'École biblique de Jérusalem, sous la dir. de Jean-Michel Poffet, Paris, Les Éd. du Cerf, 2002.

L'autorité du passé dans les sociétés médiévales, Actes du colloque, Rome, 2-4 mai 2002, organisé par l'Institut historique belge de Rome; l'École française de Rome; l'Université libre de Bruxelles; l'Université Charles de Gaulle-Lille 3, sous la dir. de Jean-Marie SANSTERRE, Rome, École française de Rome (Collection de l'École française de Rome, 333), Paris, diff. de Boccard, 2004.

Les actes comme expression du pouvoir au haut Moyen Âge, Actes de la table ronde de Nancy, 26-27 novembre 1999, éd. par Marie-José GASSE-GRANDJEAN et Benoît-Michel TOcK, Turnhout, Brepols, 2003.

BeLting, Hans, Pour une anthropologie des images, Paris, 2005.

BERTHELOT, Anne, Figures et fonctions de l'écrivain au XIII siècle, Paris, Vrin, 1991.

BOUREAU, Alain, «Les cérémonies royales françaises entre performance juridiques et compétences linguistiques ", Annales ESC, 54, 1991/4, pp. 1265-1333.

BOURRICAUD, François, Esquisse d'une théorie de l'autorité, Paris, 1961.

BOUTET, Dominique, Charlemagne et Arthur ou le roi imaginaire, Paris, Champion, 1992. 
CERQUIGLINI-TOULET, Jacqueline, «Fama et les preux: nom et renom à la fin du Moyen Âge ", Médiévales, 24 (1993), pp. 35-44.

CHALMERS, Thomas, The evidence and authority of the christian revelation, Bristol, Thoemmes Press, 2000.

COMPAGNON, Antoine, Qu'est-ce qu'un auteur?, cours en ligne (http://www.fabula.org/ compagnon/auteur.php), notamment »Quatrième leçon:Généalogie de l'autorité » (http://www.fabula.org/compagnon/auteur4.php).

CONSTABLE, Giles, "The Authority of Superiors in the Religious Communities ", dans La Notion d'autorité au Moyen Âge: Islam, Byzance, Occident, Colloques internationaux de La Napoule, session des 23-26 octobre 1978, organisés par George Makdisi, Dominique Sourdel et Janine Sourdel-Thomine, Paris, Presses Universitaires de France, 1982, pp. 189-210.

CONTAMINE, Philippe, Des pouvoirs en France: 1300-1500, Paris, Presses de l'École normale supérieure, 1992.

CORNIER, Jean-Philippe, Monnaies médiévales, reflets du pouvoir, Paris, 1996.

Culture et idéologie dans la genèse de l'État moderne, Actes de la table ronde, Rome, 15-17 octobre 1984, organisée par le Centre national de la recherche scientifique et l'École française de Rome, Rome, École française de Rome, 1985 (Collection de l'École française de Rome, 82).

DAVY, Gilduin, Le duc et la loi. Héritages, images et expressions du pouvoir normatif dans le duché de Normandie, des origines à la mort du Conquérant (fin du IXe siècle - 1087), préf. de Albert Rigaudière, Paris, De Boccard, 2004.

Les élites du pouvoir et la construction de l'État en Europe, sous la dir. de Wolfgang REINHARD, trad. de l'anglais par Hélène Aji, texte établi par Robert Descimon, Paris, Presses Universitaires de France, 1996.

L'État ou le roi : les fondations de la modernité monarchique en France, XIV ${ }^{e}-\mathrm{XVII}{ }^{e}$ siècles, Table ronde du 25 mai 1991 à l'École normale supérieure, Paris, organisée par Neithard Bulst et Robert Descimon, textes réunis par Neithard Bulst, Robert Descimon et Alain Guerreau, Paris, Éd. de la Maison des sciences de l'homme, 1996.

The Experience of Power in Medieval Europe, 950-1350: Essays in Honor of Thomas N. Bisson, edited by Robert F. Berkhofer III, Alan Cooper and Adam J. Kosto, Aldershot, Burlington (Vt.), Ashgate, 2005.

FELLER, Laurent, L'Église et la société en Occident : pouvoir politique et pouvoir religieux du VII au XI siècle, Paris, SEDES, 2001.

Foi chrétienne et églises dans la société politique de l'Occident du Haut Moyen Âge : IVe-XII siècle, Actes des XXIII ${ }^{e}$ Journées d'histoire du droit, Limoges, 2004, textes réunis par Jacqueline Hoareau-Dodinau et Pascal Texier, Limoges, Pulim, 2004.

FRAENKEL, Béatrice, La signature, genèse d'un signe, Paris, 1992. GIBERT, André, Autorité de la Bible, Valence, Bibles, 1994.

The Gowth of Authority in the Medieval West, Selected proceedings of the International Conference, Groningen, 6-9 november 1997, ed. by Martin Gosman, Arjo Vanderjagt \& Jan Veenstra, Groningen, E. Forsten, 1999, (Mediaevalia Groningana; 25).

GUENÉE, Bernard, L'Occident aux XIVe et XV $V^{e}$ siècles. Les États, 3e éd. mise à jour, Paris, Presses universitaires de France, 1987.

GUILLoT, Olivier, Arcana imperii, Limoges, Pulim, 2003.

GUILLOT, Olivier, «Le concept d'autorité dans l'ordre politique français issu de l'an mil », dans La Notion d'autorité au Moyen Âge : Islam, Byzance, Occident, Colloques internationaux 
de La Napoule, session des 23-26 octobre 1978, organisés par George Makdisi, Dominique Sourdel et Janine Sourdel-Thomine, Paris, Presses Universitaires de France, 1982, pp. 127-140.

HARSGOR, Michael, Un très petit nombre: des oligarchies dans l'histoire de l'Occident, Paris, Fayard, 1993.

L'Image, fonctions et usages des images dans l'Occident médiéval, Actes du $6^{\mathrm{e}}$ "International Workshop on Medieval Societies » (Centre Ettore Majorana, Erice, 17-23 octobre 1992), sous la direction de Jérôme BASCHET et Jean-Claude schMITT, Paris, Le Léopard d'or, 1996 (Cahiers du Léopard d'or, 5).

$\mathrm{JACOB}$, Robert, Image de justice, essai sur l'iconographie judiciaire du Moyen Âge à l'Âge Classique, Paris, 1994.

KANTOROWICZ, Ernst Hartwig, Laudes regiae : une étude des acclamations liturgiques et du culte du souverain au Moyen Âge, trad. de l'anglais par Alain Wijffels, Paris, Fayard, 2004.

KoJÈVE, Alexandre, La notion de l'autorité, Paris, 2003.

KRYNEN, Jacques, L'empire du roi : idées et croyances politiques en France, XIII-XV siècle, Paris, Gallimard, 1993.

KRYNEN, Jacques, Idéal du prince et pouvoir royal en France à la fin du Moyen Âge : 1380-1440 : étude de la littérature politique du temps, Paris, A. et J. Picard, 1981.

KUTTNER, Stephan, «On «Auctoritas» in the Writing of Medieval Canonists: the Vocabulary of Gratian », dans La Notion d'autorité au Moyen Âge : Islam, Byzance, Occident, Colloques internationaux de La Napoule, session des 23-26 octobre 1978, organisés par George Makdisi, Dominique Sourdel et Janine Sourdel-Thomine, Paris, Presses Universitaires de France, 1982, pp. 69-82.

LECLERC, Gérard, Histoire de l'autorité : l'assignation des énoncés culturels et la généalogie de la croyance, Paris, Presses Universitaires de France, 1996.

MINNIS, A. J., Medieval Theory of Authorship: Scholastic Literary Attitudes in the Later Middle Ages, Londres, Scholar Press, 1972.

MINNIS, A. J., et Scott, A. B., Medieval Literary Theory and Criticism, Oxford, Clarendon Press, 1988.

PACAUT, Marcel, Doctrines politiques et structures ecclésiastiques dans l'Occident médiéval, London, Variorum reprints, 1985.

PASTOUREAU, Michel, Figures et couleurs, Etudes sur la symbolique et la sensibilité médiévales, Paris, \$, 1986.

PAStoureau, Michel, Les sceaux, Typologie des sources du Moyen Âge occidental $n^{\circ} 36$, Turnhout, 1987.

Penser le pouvoir au Moyen Âge: $\mathrm{VIII}^{e}-\mathrm{XV}{ }^{e}$ siècle, Etudes d'histoire et de littérature offertes à Françoise Autrand, textes réunis par Dominique BOUTET et Jacques VERGER, Paris, Éditions de la rue d'Ulm, 2000.

Per me reges regnant: la regalità sacra nell'Europa medievale, a cura di Franco CARDINI, Maria SALTARELLI, Rimini, Ed. Il cerchio / Siena, Ed. Cantagalli, 2002.

POPESCU, Dan-Alexandru, Dei gratia... : sacre et couronnement médiéval : Moyen Âge en France et Moyen Âge dans les principautés roumaines (VIII ${ }^{e}-\mathrm{XVIII}{ }^{e}$ siècle), avant-propos par Bernard Merdrignac, Sibiu, Continent, 2002.

Le pouvoir au Moyen Âge: idéologies, pratiques, représentations (Séminaire de l'équipe de recherches Sociétés, idéologies et croyances au Moyen Âge), sous la dir. de Claude CAROZZI et Huguette TAVIANI-CAROZZI, Aix-en-Provence, Publications de l'Université de Provence, 2005. 
Les Pouvoirs de commandement jusqu'à 1610, Actes du 105e Congrès national des sociétés savantes, Caen, 1980, (Section de philologie et d'histoire jusqu'à 1610, Paris, Comité des travaux historiques et scientifiques, 1984).

Power of the weak. Studies on medieval women, ed. by Jennifer Carpenter and Sally-Beth MacLean, Urbana (Ill.), Chicago (Ill.), University of Illinois Press, 1995.

Les princes et le pouvoir au Moyen Âge, Actes du XXIIIe Congrès de la SHMESP [Société des historiens médiévistes de l'enseignement supérieur public], Brest, mai 1992, organisé avec le concours du Centre de recherche bretonne et celtique, Paris, Publications de la Sorbonne, 1993 (Publications de la Sorbonne. Série Histoire ancienne et médiévale, 28). La propaganda politica nel basso Medioevo, Atti del XXXVIII Convegno storico internazionale, Todi, 14-17 ottobre 2001, Centro italiano di studi sul basso medioevo, Accademia Tudertina, Centro di studi sulla spiritualità medievale dell'Università degli studi di Perugia, Spoleto, Centro italiano di studi sull'alto medioevo, 2002.

The Propagation of Power in the Medieval West, Selected proceedings of the International conference, Groningen, 20-23 November 1996, ed. by Martin Gosman, Arjo Vanderjagt \& Jan Veenstra, Groningen, E. Forsten, cop. 1997 (Mediaevalia Groningana ; 23).

RAYNAUD, Christiane, Images et pouvoirs, Paris, Le Léopard d'or, 1993 (76-Le Havre : Impr. SNAG).

Représentation, pouvoir et royauté à la fin du Moyen Âge, Actes du colloque organisé par l'Université du Maine, les 25 et 26 mars 1994, éd. par Joël BLANCHARD, postf. de Philippe Contamine, Paris, Picard, 1995.

SASSIER, Yves, Structures du pouvoir, royauté et Res publica: France, IX ${ }^{e}-\mathrm{XII}{ }^{e}$ siècles, MontSaint-Aignan, Publications de l'Université de Rouen, 2004.

Seigneurs et seigneuries au Moyen Âge, Actes du 117e Congrès national des sociétés savantes, Clermont-Ferrand, 1992, Section d'histoire médiévale et de philologie, Paris, Ed. du CTHS, 1993.

SHEPARD, Laurie, Courting Power: Persuasion and Politics in the Early Thirteenth Century, New York, London, Garland, 1999.

SMITH, Susan L., The Power of Women : A Topos in Medieval Art and Literature, Philadelphia, University of Pennsylvania press, 1995.

STROLL, Mary, Symbols as power: the papacy following the investiture contest, Leiden, E. J. Brill, 1991.

Topographies of power in the early Middle Ages, ed. by Mayke de Jong and Frans Theuws with Carine van Rhijn, Leiden, Brill, 2001 (The transformation of the Roman world : a scientific programme of the European science foundation, 6).

VAUCHEZ, André, Saints, prophètes et visionnaires : le pouvoir surnaturel au Moyen Âge, Paris, Albin Michel, 1999.

Vincennes aux origines de l'État moderne, Actes du colloque scientifique de Vincennes, les 8, 9 et 10 juin 1994, organisé par Jean Chapelot et Élisabeth Lalou, Paris, Presses de l'École normale supérieure, 1996.

WERNER, Karl Ferdinand, Naissance de la noblesse : l'essor des élites politiques en Europe, Paris, Fayard, 1998. 
INDEX

Mots-clés : autorité, figure, mythe, royauté, pouvoir, politique, littérature

Keywords : authority, figure, myth, kingship, power, politics, literature 